\title{
Navigational control of bacteria: the design of a synthetic chemotactic biological system
} James Brown $* 1,2$ Address: ${ }^{1}$ Department of Plant Science, University of Cambridge, Cambridge, CB2 3EA, UK and ${ }^{2}$ Department of Engineering, University of
Cambridge, Cambridge, CB2 31PZ, UK

Email: James Brown* - jrb62@cam.ac.uk

* Corresponding author

from BioSysBio 2007: Systems Biology, Bioinformatics and Synthetic Biology

Manchester, UK. II-I 3 January 2007

Published: 8 May 2007

BMC Systems Biology 2007, I(Suppl I):SI4 doi:I0.I I86/I752-0509-I-SI-SI 4

This abstract is available from: http://www.biomedcentral.com//752-0509/I?issue=SI

(c) 2007 Brown; licensee BioMed Central Ltd.

\section{Background}

Synthetic Biology is a rapidly developing field, which sees engineering principles applied to natural biological systems with a view to re-engineer them for useful purposes. This study focused on chemotaxis, the natural directed motion of a micro-organism toward environmental conditions it deems attractive, with an aim of demonstrating external navigational control over $E$. coli bacteria. The concept has potential long-term applications in a range of fields, including biological and environmental sensors, drug discovery and the maintenance or enhancement of human health.

\section{Method}

The periplasmic maltose-binding protein (MBP) is essential for maltose transport and taxis. It was placed under external control via synthetic plasmids containing 2 different IPTG-based promoters coupled to the MBP-encoding malE and malE31 [1] genes. These were transformed into three engineered $\triangle$ malE E. coli strains allowing for both permanent and temporary reactivation of maltose chemotaxis. The stochastic simulator StochSim [2] was adapted from the standard aspartate model to consider the associated maltose regulon.

\section{Results}

The re-engineering of the natural chemotaxis system was successfully demonstrated on both the macro and micro scale using swimming and agarose plug assays. It was shown maltose chemotaxis could be initiated and repressed by an external signal. Stochsim was successfully adapted to model the maltose system, with simulation data for the adaptation and dependence on MBP shown to closely match experimental data [3].

\section{Conclusion}

A novel approach demonstrated the feasible control of the sensory system and resultant motile behaviour of synthetically engineered $E$. coli cells. This provides an appropriate tool for generating experimental data that in turn may feed the computational modelling of chemotaxis. This allows a better understanding of the natural system and how one might go about specifically engineering that system for a useful cause.

\section{References}

I. Sandén AM, Boström M, Markland K, Larsson G: Solubility and proteolysis of the Zb-MalE and Zb-MalE3 I proteins during overproduction in Escherichia coli. Biotechnol Bioeng 2005, 90:239-247.

2. Morton-Firth C], Shimizu TS, Bray D: A free-energy-based stochastic simulation of the tar receptor complex. J Mol Biol I999, 286:1059-1074.

3. Manson MD, Boos W, Bassford PJ Jr, Rasmussen BA: Dependence of maltose transport and chemotaxis on the amount of maltose-binding protein. J Biol Chem 1985, 260:9727-9733. 